\title{
Frontières
}

\section{Les risques de violence dans l'accompagnement}

\section{Jean-François Malherbe}

Volume 17, numéro 1, automne 2004

\section{Au péril de l'accompagnement}

URI : https://id.erudit.org/iderudit/1073599ar

DOI : https://doi.org/10.7202/1073599ar

Aller au sommaire du numéro

Éditeur(s)

Université du Québec à Montréal

ISSN

1180-3479 (imprimé)

1916-0976 (numérique)

Découvrir la revue

Citer cet article

Malherbe, J.-F. (2004). Les risques de violence dans l'accompagnement.

Frontières, 17(1), 8-14. https://doi.org/10.7202/1073599ar

\section{Résumé de l'article}

Les pratiques cliniques propres aux soins palliatifs comportent des risques non négligeables de violence. Le regard médical sur la crise existentielle majeure que les soins palliatifs tentent de soulager consiste trop souvent à réduire un drame existentiel à ses dimensions corporelles. Il est moins difficile, en effet, d'orienter les décisions cliniques à partir de mesures biologiques objectives qu'à partir de la subjectivité des mourants. C'est dans cette radicale réduction du sujet à l'objet que se manifeste le risque de violence dans les soins palliatifs. Dans ce contexte, les soignants comme les soignés se perçoivent comme des rouages d'un mécanisme déshumanisant et se considèrent déchargés de toute responsabilité personnelle à l'égard du processus anonyme qui les accable. Pour pallier cette déviation paradoxale des soins palliatifs, nous proposons une approche éthique de la violence fondée sur une élucidation de la signification subjective de la mort.
Ce document est protégé par la loi sur le droit d'auteur. L'utilisation des services d’Érudit (y compris la reproduction) est assujettie à sa politique d'utilisation que vous pouvez consulter en ligne.

https://apropos.erudit.org/fr/usagers/politique-dutilisation/ 


\section{Résumé}

Les pratiques cliniques propres aux soins palliatifs comportent des risques non négligeables de violence. Le regard médical sur la crise existentielle majeure que les soins palliatifs tentent de soulager consiste trop souvent à réduire un drame existentiel à ses dimensions corporelles. Il est moins difficile, en effet, d'orienter les décisions cliniques à partir de mesures biologiques objectives qu'à partir de la subjectivité des mourants. C'est dans cette radicale réduction du sujet à l'objet que se manifeste le risque de violence dans les soins palliatifs. Dans ce contexte, les soignants comme les soignés se perçoivent comme des rouages d'un mécanisme déshumanisant et se considèrent déchargés de toute responsabilité personnelle à l'égard du processus anonyme qui les accable. Pour pallier cette déviation paradoxale des soins palliatifs, nous proposons une approche éthique de la violence fondée sur une élucidation de la signification subjective de la mort.

Mots clés: éthique clinique - soins palliatifs - violence - crise existentielle subjectivité - mort.

\section{Abstract}

The occurrence of violence is a true risk within the clinical relations involved in palliative care. The medical approach of the human crisis in which occurs the necessity of palliative care implies a drastic reduction of the existential dimensions of that crisis. The measurable biological aspects of the crisis are taken under consideration more easily than the "subjective" ones. Violence occurs in such a subject-destructive reduction. In that context, the caring human beings as well as the cared ones feel themselves as single wheels involved in a mechanical dishumanizing process. And therefore they consider themselves as discharged of any personal liability in this anonymized process. In order to palliate this paradoxal derivation of palliative care, we propose a renewed approach of violence phenomena in clinical contexts. This ethical approach is grounded on a philosophical elucidation of the subjective meaning of the dying process.

Key words: clinical ethics - palliative care - violence - existential crisis subjectivity - death.

\section{LES RISQUES \\ DE VIOLENCE DANS L'ACCOMPAGNEMENT'}

\section{Jean-François Malherbe, Ph.D., professeur titulaire, directeur de la Chaire d'Éthique appliquée, Université de Sherbrooke.}

Les risques de violence dans les relations d'aide et en particulier dans les soins palliatifs ne sont pas négligeables. Pour les situer à leur juste place, il est nécessaire de les mettre dans une perspective d'ensemble qui fera apparaître à la fois leur réalité et les moyens de les éviter.

\section{LES «SOINS PALLIATIFS » ENTRE L'ACHARNEMENT ET L'EUTHANASIE}

Lorsque je travaillais comme conseiller en éthique dans un service de soins intensifs, je fus appelé par un des médecins du service qui était confronté, à propos d'une de ses patientes, à une altercation vive entre les deux filles de celle-ci. La cadette reprochait à l'aînée de vouloir faire souffrir sa mère et, inversement, l'aînée soupçonnait sa sœur de vouloir tuer sa mère pour «en finir» avec ces maudites souffrances. En dépit de son expérience pourtant bien enracinée, le médecin était hésitant sur la conduite à tenir face à ces deux femmes et me demandait mon avis.

Je proposai aux deux filles de la patiente de reprendre leur souffle et de s'asseoir. Puis je leur suggérai d'exprimer ce qu'elles souhaitaient pour leur mère plutôt que de s'obstiner chacune dans son refus de ce qu'elle soupçonnait l'autre de vouloir. Au bout de quelques minutes de conversation, il est apparu assez clairement que l'une comme l'autre reconnaissaient que leur maman s'approchait de la fin de son chemin avec elles et que toutes deux souhaitaient qu'elle puisse les quitter paisiblement, sans souffrance. Elles exprimaient également leur espoir que la sérénité de leur maman les aiderait à accepter la séparation qui désormais leur apparaissait inéluctable.

Face à cet unisson, que l'altercation qui les opposait quelques minutes plus tôt ne laissait pas prévoir, je les invitai à tenter d'exprimer ce qui les avait mis en colère au point d'attribuer à l'autre le désir de ce que chacune craignait. La cadette expliqua que le frère d'une de ses amies, qui avait été victime d'un très grave accident de la route, avait souffert un épouvantable calvaire avant de mourir corseté par mille et une machines à l'aide desquelles les médecins avaient tenté de l'obliger à vivre. De son côté, l'aînée gardait le souvenir tenace du drame qu'avaient suscité dans la famille les bruits qui avaient circulés autour de la mort d'un de leurs oncles, précisément le frère aîné de leur maman, qui, disaient certains, avait été envoyé «ad patres»sans ménagements par des médecins qui n'avaient pas pris le temps de s'occuper attentivement de son cas.

Acharnement thérapeutique ou euthanasie? Peu m'importe ici de savoir si ces deux femmes avaient ou non une vision déformée de ce que les médecins avaient fait en réalité. Il n'y a nulle place dans mes propos pour aucun procès d'intention. Ce qui m'importe, au contraire, c'est de 
souligner la symétrie des deux pratiques excessives que représentent à mes yeux l'acharnement thérapeutique et l'euthanasie. L'une des pratiques consiste pour ainsi dire à "avancer» l'heure de la mort et l'autre à la «retarder». Or, c'est précisément entre précipitation et hésitation face à la mort qui vient, précipitation et hésitation qui culminent toutes deux dans la violence, que se dessine l'espace dans lequel prend tout son sens le projet des soins palliatifs que je caractériserais volontiers en disant qu'ils visent à laisser la mort «venir à son heure», aussi paisiblement que possible.

\section{LA NÉCESSAIRE OBJECTIVATION MÉDICALE DU SUJET ET SES LIMITES}

Cela dit, il convient de ne pas simplifier outrancièrement les exigences des pratiques cliniques à l'approche de la mort. Le point de vue que je souhaite illustrer par mes propos ne s'inscrit aucunement dans la perspective d'un quelconque mouvement anti-science. Tout au contraire. Il s'agit d'apprécier à leur juste valeur les immenses progrès réalisés par la médecine intensiviste et les disciplines voisines, et de les saluer. Mais précisément, pour les apprécier à leur juste valeur, il convient de s'interroger sur les présuppositions méthodologiques qui leur ont ouvert la voie vers de tels succès.

C'est en jetant sur l'être humain un regard très particulier que les sciences biomédicales ont pu atteindre les performances cliniques que vous connaissez. Je qualifierais ce regard d'objectivant. C'est en considérant l'humain comme un corps-machine que la médecine bernardienne, héritière directe de la méthode cartésienne, s'est ménagé la voie vers le succès opératoire. Le regard biomédical abstrait l'organisme de la personne, il découpe mentalement la chair vécue qu'habite le sujet humain pour en extraire cette machine cybernétique qui constitue son objet d'étude. Ce n'est pas d'abord l'histoire personnelle d'une existence vécue qui retient l'attention de l'homme de science, ce sont les mécanismes, à vrai dire très complexes, qui lui servent de support matériel.

Ce regard objectivant est infiniment précieux. C'est, en effet, la conquête de cette approche scientifique de l'humain qui a permis le développement d'une science exacte de l'organisme qu'il habite. Mais il ne faudrait pas que ce moment nécessaire de la méthode médicale, le moment de l'objectivation de l'humain, reste le seul point de vue possible sur l'humain. Cette exclusivité serait en effet par trop réductrice. L'humain est une machine cybernétique, certes. Et à ce titre, il est susceptible d'une analyse mécanique des dysfonctionnements qui l'affectent. Mais il n'est pas qu'une machine cybernétique. Il n'est pas qu'un corps objet de science. Il est aussi un sujet. Le sujet de sa propre existence vécue. Et c'est en définitive ce sujet que la médecine a pour finalité de servir, et non pas la seule machine cybernétique que la biologie lui apprend à prendre en considération et qu'elle est devenue extrêmement habile à entretenir et à réparer. Il s'agit pour le clinicien qui veut être un thérapeute et pas seulement un ingénieur biomédical de veiller dans chacune de ses décisions à mettre sa science de la machine humaine au service de celui qui habite cette machine.

Pour le dire simplement, je pense que c'est la prise en considération trop exclusive de la machine humaine, et la griserie que peuvent provoquer les succès mécaniques de cette approche scientifique, qui induit les plus brillants médecins en tentation de scientisme. C'est-à-dire à succomber à l'illusion que la science suffit à résoudre tous les problèmes qui se présentent à eux. Je voudrais attirer votre attention sur le fait que l'acharnement thérapeutique comme l'euthanasie sont deux formes de violence qui découlent de cette ivresse scientiste. La science qui perd la conscience de ses propres limites se mue en idéologie mortifère. Au contraire, la science qui s'accompagne de la conscience vive et active de ses propres limites reste au service de l'humain sans se laisser emporter par l'impression de toute-puissance que risque toujours d'induire le succès technique. La pratique des soins palliatifs, c'est la concrétisation de cette science médicale qui reste vigilante sur ses propres limites et ne perd pas de vue sa propre finalité. C'est un projet qui mobilise toutes les connaissances scientifiques médicales au service des sujets humains qui atteignent, comme cela arrivera à chacun d'entre nous, le bout de leur chemin parmi leurs semblables. C'est un projet de nonviolence en fin de vie.

Accomplir leur tâche dans une telle perspective est un véritable défi pour les médecins car ils auront non seulement à réfléchir pour ainsi dire philosophiquement à leur propre pratique - et il faut reconnaître que jusqu'ici, les facultés de médecine les ont rarement bien équipés à cet égard -, mais aussi à affronter leurs patients qui le plus souvent leur demandent de résoudre leurs problèmes de façon pour ainsi dire mécanique, c'est-à-dire en leur évitant soigneusement de devoir s'impliquer personnellement dans cette solution qui les concerne pourtant au premier chef.

\section{L'INAUDIBLE PAROLE DU SUJET EN CRISE}

Loin des violences médicales qui résultent encore trop souvent du scientisme prométhéen, les soins palliatifs tentent de laisser les humains mourir aussi sereinement que possible «à leur heure ». Mais cette voie n'est pas un chemin de facilité. C'est tout au contraire une entreprise difficile, toute en nuances, délicate. Il ne s'agit plus de mécanique scientifique mais de dentelle clinique. Il s'agit de travailler au cas par cas, de produire du cousu main, de s'inscrire aussi harmonieusement que possible dans la trajectoire singulière d'une vie pour que la courbe en rejoigne paisiblement l'asymptote.

Et dans cette entreprise généreuse et attentive, une place toute particulière est réservée, autant que faire se peut, à la parole de la personne qui bénéficie des soins attentifs que leur prodiguent les équipes de soins palliatifs. "Palliatif» s'entend ici dans un sens bien précis. Il ne s'agit plus de vouloir guérir, ce qui est la légitime logique des soins «curatifs», mais bien de "pallier» les inconforts, les malaises, les mal-être aussi, que peut vivre notre semblable à la fin de son chemin parmi nous. Évidemment, le point d'inflexion qui sépare, sur la courbe d'une vie, la portion antérieure au cours de laquelle ne pas prodiguer les éventuels soins curatifs nécessaires constituerait une coupable négligence, de la portion ultérieure au cours de laquelle il serait tout aussi coupable de ne pas céder le pas aux soins palliatifs, n'est pas toujours facile à déterminer. Il n'empêche que ce point existe et je veux croire que c'est notamment dans le dialogue le plus ouvert entre intensivistes et palliativistes que pourra s'acquérir progressivement l'expérience médicale la plus pertinente à ce sujet.

Mais précisément, ce point est particulier à chaque cas. C'est pourquoi sa détermination ne peut s'effectuer correctement sans la participation, autant que faire se peut, de la personne la plus concernée. Et, en effet, pour cette dernière, atteindre ce point et le reconnaître signifie l'acceptation de la mort qui vient. Il est rare que le consentement à quitter tout ce à quoi on a pu tenir et à quoi on tient sans doute encore toujours, soit donné d'emblée. La raison en est que chacun de nous se construit de sa propre vie un récit dans lequel il est rare que la mort ait déjà sa juste place. À chacune des grandes étapes de sa vie, le sujet humain connaît des crises c'est-à-dire, comme l'indique l'étymologie grecque du mot (krisis), de ces moments de discernement qui sont l'occasion de reconnaître ou de donner un sens à sa vie. Et, de crise en crise, l'humain s'achemine vers la crise ultime que provoque l'approche de la fin. Cette dernière crise, il convient, autant que possible, qu'elle puisse venir au langage. Ou plus exactement: il ne conviendrait absolument pas qu'elle soit empêchée de venir au langage si le sujet qui la vit s'efforce de la dire. 
Mais nul d'entre nous n'aime parler à des murs. Tous nous apprécions la présence chaleureuse d'une oreille attentive lorsque nous sommes pour ainsi dire affrontés au travail du sens dans la crise que nous traversons. C'est dire le caractère indispensable de ce que l'on est convenu aujourd'hui d'appeler «l'accompagnement». Qu'il soit le fait de bénévoles, de professionnels des soins palliatifs ou de proches parents ou amis importe, mais il importe encore bien plus qu'une telle oreille soit disponible et disposée à entendre. Il existe encore trop de cas dans lesquels la violence médicale de traitements disproportionnés ou inutiles, ou même de la pratique euthanasique, exclue avec force toute occasion que cette parole puisse se dire et qu'elle soit entendue. C'est inacceptable car au travers de ces ultimes expressions, c'est souvent une naissance qui se travaille, un avènement qui se trame, un passage d'être qui s'accomplit. Et nul d'entre nous n'a le droit d'empêcher que l'autre naisse avant que de mourir.

\section{LES CHEMINS SINUEUX DE LA VÉRITÉ}

Lorsque j'étais professeur d'éthique à la Faculté de médecine de l'Université de Louvain, mes étudiants me demandaient souvent s'il fallait «vraiment dire la vérité» aux patients. Chaque fois, je tentais de leur faire prendre conscience de l'arrière-plan épistémologique de leur question. Quelle était leur précompréhension de la vérité à dire ou à ne pas dire ou à ne dire qu'à moitié? Il me paraissait que leur discours s'organisait comme si une vérité était contenue dans leur savoir comme un liquide dans un cruchon et que leur question portait sur l'opportunité, ou le devoir de déverser une quantité plus ou moins grande de liquide de leur cruchon dans le verre de leur patient. Mais qu'est-ce que la vérité? Ponce Pilate a rendu célèbre cette difficile question à laquelle nous n'avons toujours pas répondu convenablement pour ce qui concerne le domaine médical.

Pour ma part, je faisais observer à mes étudiants que la vérité qu'ils savaient était de nature statistique et concernait des populations tandis que la vérité qui préoccupait éventuellement leurs patients était de nature particulière et ne concernait que leurs cas singuliers. Ce qui préoccupe un patient, ce n'est pas la statistique, c'est son pronostic. Ce que sait un médecin, c'est un diagnostic. Et le pronostic qu'il peut risquer reste toujours essentiellement aléatoire. Certes, lorsqu'il a une longue expérience clinique, le médecin parvient à resserrer quelque peu la marge d'approximation que contient toujours un pronostic établi sur une base statistique. Il n'empêche que, dans son principe, une incertitude toujours demeure et que le patient s'en accommodera mal. Ici encore, le risque de violence est patent.

Dès lors, je répondais ceci aux étudiants: la vérité n'est pas un contenu à déverser du cruchon de votre science dans le verre d'ignorance de vos patients. La vérité, c'est un événement qui survient entre deux personnes qui se risquent à se parler aussi respectueusement que clairement. Et j'ajoutais à ma réponse ce commentaire en forme de recommandation. Lorsque vous rendez visite à un patient, assoyez-vous en prenant garde que vos yeux et les siens soient à la même hauteur. Dites-lui que vous venez prendre de ses nouvelles et que vous êtes là pour répondre à ses questions. Écoutez les questions et les inquiétudes, répondez clairement, sans jamais mentir, et laissez toujours ouverte la porte de la prochaine question. Votre patient mesure mieux que vous ce qu'il est capable d'entendre. Il ne posera pas la question qui le confronterait à une réponse qui lui ferait trop mal. Mais demain peut différer d'aujourd'hui et il est des résistances qui se transforment en ouverture, des refus qui se transmutent en assentiments, des dénis qui se dissolvent. Il arrive aussi que l'enfermement soit tenace. La vérité, c'est la parole de vie qui ne fait pas de concession au mensonge. L'éthique en fin de vie, c'est la recherche de cette vérité-là. De cette vérité qui nous permet d'achever de naître avant que de mourir.

\section{L'HUMAINE CONDITION DES} SOIGNÉS COMME DES SOIGNANTS

Pour qu'une telle vérité puisse advenir, il est des conditions à mettre en place. Cellesci concernent tout aussi bien les soignants que les soignés et, en un sens que je vais préciser sans tarder, elles sont identiques des deux côtés de la relation de soin. C'est qu'il s'agit, en définitive, du côté des soignants comme des soignés, d'assumer l'humaine condition.

Mais comment caractériser en quelques mots l'humaine condition sans tomber dans le poncif ou la caricature? Peut-être y a-t-il des indications à recueillir d'une voie que j'ai explorée par ailleurs (Malherbe, 1987)? J'avais tenté de construire un concept d'autonomie conçu précisément comme assomption de l'humaine condition, que j'avais décrite, à la suite de toute une tradition philosophique, à l'aide des trois concepts de «solitude», «finitude» et «incertitude». Pour illustrer ces concepts, je partirai de cette possible conversation entre un médecin et son patient que je viens d'évoquer et où il était question de la «vérité».

Si le médecin est relativement certain des connaissances statistiques qu'il met régulièrement à jour, il reste radicalement

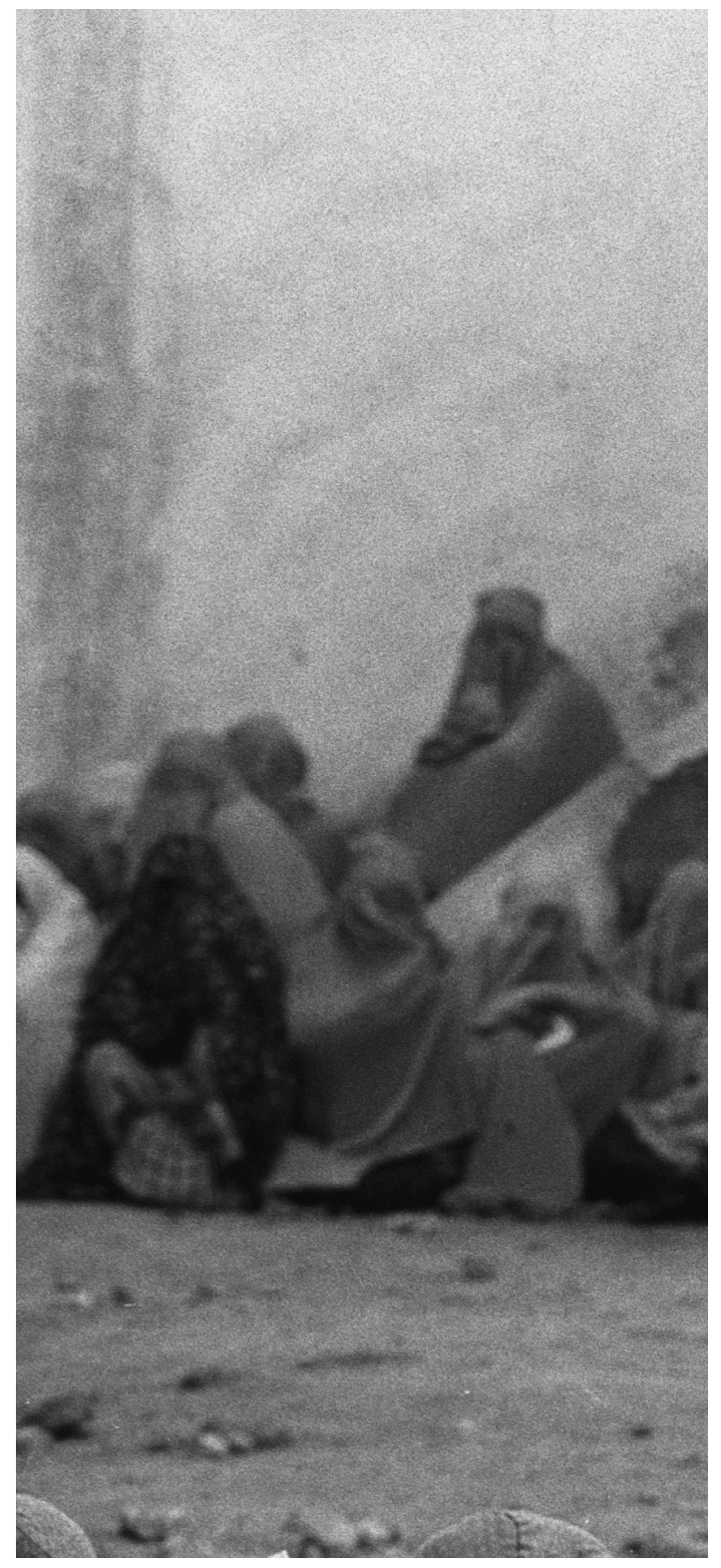

Taliban, Afghanistan

incertain de l'avenir concret de son patient. Cette «incertitude» est radicale en ce sens que nulle donnée supplémentaire ne pourrait la combler, hormis évidemment la mort du patient elle-même. Assumer cette incertitude radicale n'est pas seulement une qualité du clinicien. C'est aussi un défi auquel est exposé le patient qui, jusqu'à la fin, restera incertain du détail de son sort. C'est, en un mot, un trait essentiel de toute existence humaine. Et, si nous voulons vraiment naître à nous-mêmes avant que de mourir, il nous faut apprendre à vivre avec notre radicale incertitude.

Il en va de même, mutatis mutandis, pour notre «finitude». Qu'est-ce à dire? On pourrait en illustrer la rigueur en rappelant la distance infranchissable qui sépare le désir du médecin de sauver son patient et les limites concrètes de son art 
Je compare volontiers l'aidant(e) à une sage-femme ou à un obstétricien et l'aidé(e) à une parturiente. Je serais même tenté, si cela ne paraissait pas trop saugrenu, de mettre ce dernier substantif au masculin: un «parturient». Tant il est vrai que donner naissance à soi-même est une tâche essentielle qui ne fait pas acception du sexe du sujet concerné.

En ce sens, la relation humaine fondamentale est une relation d'aide. Elle participe de ce travail par lequel, tour à tour, parturiente et obstétricien, nous nous donnons naissance à nous-mêmes dans la réciprocité. Plus précisément, on pourrait dire que la relation humaine fondamentale est faite de deux relations d'aide réciproques.

La «relation d'aide» est un cas particulier de cette relation fondamentale. Sa particularité consiste en ceci qu'elle est asymétrique: il y a un sujet qui demande de l'aide et un autre qui la lui accorde au titre d'un statut particulier: parent, éducateur, soignant, compagnon de route, etc.

Dans le domaine de la santé, une relation d'aide a pour but de réinvestir subjectivement le "corps machine» du sujet (c'est-à-dire le «corps qu'il $\boldsymbol{a}$ »), de le soutenir à habiter le «corps qu'il est». C'est, en définitive, d'accompagner le sujet, jusqu'à ce qu'il «en sorte», dans la maladie qu'il vit nécessairement - même si ce n'est qu'implicitement - comme une profonde crise personnelle.

\section{LA MALADIE \\ COMME CRISE SUBJECTIVE}

La médecine, nous disent les dictionnaires, est «L'art de soigner et de prévenir les maladies de l'homme». Mais qu'est-ce qu'une maladie? C'est, toujours selon le dictionnaire: «Une altération organique ou fonctionnelle considérée dans son évolution, et comme une entité définissable.» Comme toujours dans les dictionnaires, on reste prisonnier de la clôture du langage. Pour en sortir, il est un moyen radical: en revenir à la perception subjective de la maladie.

Et, pour le sujet, la maladie n'est-elle pas le plus souvent vécue comme une crise? Et n'est-elle pas même parfois une réponse à une crise? Il est hors de question de vouloir définir ici la crise dans tous ses aspects économiques, sociaux, symboliques et psychologiques, mais je voudrais tenter d'élucider le concept de crise afin qu'il éclaire, lui aussi, une des nombreuses facettes de ce que nous appelons "maladie». Il s'agira moins de définir la crise que de disposer quelques phrases pour éclairer la question.

Je parle d'une crise d'asthme, d'une crise d'appendicite, d'une crise de foie ou d'une crise de larmes pour désigner quelque chose qui est trop fort pour moi et que je ne peux pas soigner avec mes moyens habituels. Généralement, je ne pleure pas, mais il arrive que je pleure quand le choc émotionnel est trop fort pour mes possibilités habituelles, ordinaires. Dans un autre registre, on parle d'une crise économique ou d'une crise générale. On dit souvent que notre époque est une époque de crise. Qu'est-ce que cela veut dire?

II y a un lien très étroit entre la société et l'individu dans cette question. Du point de vue social, par exemple, on dira que le chômage, l'inflation, la dévaluation et tous les autres phénomènes que nous connaissons sont les signes que notre société est en crise dans son organisation, dans ses buts et dans ses moyens. Chaque fois qu'il y a crise surgit une question touchant au sens et à l'espérance ou à l'absurde et à la désespérance. Dans la société actuelle, il y a un réel effondrement du sens et donc une réelle crise. Nous n'y pouvons sans doute pas grand-chose, mais il peut dépendre de nous que la crise soit une occasion de changement.

Nous ne savons plus à quel saint nous vouer face aux risques qui se profilent à l'horizon. Nous ne savons plus quel langage tenir sur ce qui fait notre vie. C'est là, pour moi, la véritable question: comment vivre dans cet environnement où il n'y a pas moyen de tenir un langage qui nous dise à nous-mêmes la vérité de ce que nous devenons? C'est dans ce contexte culturel, qui nous conduit à une certaine désespérance, que surviennent les crises personnelles. Bien entendu, quand on rencontre quelqu'un qui est «en crise», il s'agit le plus souvent de crises personnelles, mais celles-ci ne prennent leur sens véritable et profond que dans le contexte d'un effondrement général du sens de notre société.

En quoi consiste la crise pour l'individu? Pour un individu, être en crise, c'est perdre, ou avoir peur de perdre, l'objet dans lequel il investit tout le sens de sa vie. L'absence de cet objet fait que la vie n'a plus de sens. Cet objet peut être un métier, un amour, un enfant... Ce peut être tout ou n'importe quoi, mais pour la personne qui s'est investie à l'égard de cet objet, celui-ci est précisément tout et pas n'importe quoi. Une crise est une situation dramatique dans laquelle le sens est soit radicalement mis en cause, soit absent.

Mon intention n'est pas de dire quel est le sens de la vie. En effet, croire qu'il peut dire aux autres le sens de la vie alors que c'est à chacun qu'il revient de l'inventer, c'est la plus mauvaise chose qui puisse arriver à un philosophe. Comment pourrait-il, en effet, prétendre parler du sens de la vie alors qu'il pourrait à peine balbutier quelques mots sur le sens de la sienne?
Mais il incombe peut-être au philosophe de dire quelque chose sur la façon dont se construit le sens de la vie.

Il est intéressant de réfléchir à la parole. Quand on rencontre quelqu'un qui est «en crise», c'est généralement quelqu'un qui raconte, ou quelqu'un qui ne dit rien (mais alors son silence est lui-même éloquent), ou encore quelqu'un qui voudrait dire qui il est mais ne le sait plus. La crise est presque toujours une crise du sens. Quelqu'un se trouve dans l'impossibilité de raconter sa propre vie avec un minimum de cohérence; il ne se sent plus capable de dire pourquoi il vit (il s'agit là de la crise radicale, bien entendu). Et pourtant, c'est quand on raconte sa vie que la vie prend un sens, que le sens émerge; c'est quand on fait le récit de sa vie, le récit de sa difficulté à un ami, à soi-même ou à un «spécialiste» que le sens peut surgir. Mais, pour qu'il surgisse, il faut reconnaître clairement qu'il est absent.

Faire le récit de sa vie lui donne un sens. Mais qu'est-ce que le récit? Ce n'est pas ce qui est récité, mais ce qui est raconté. Et raconter n'est pas fabuler.

On ne raconte pas n'importe quoi quand on raconte sa vie. Et on ne la raconte pas n'importe comment, même si on ne la raconte jamais deux fois de suite de la même façon. Il est d'ailleurs impossible de la raconter deux fois de la même façon.

Il est indispensable de raconter sa vie parce que la vie est faite d'événements qui se succèdent et n'ont pas toujours de liens très clairs entre eux. Ces événements qui se succèdent et sont vécus par une même personne demandent à être unifiés. Raconter sa vie permet d'unifier la dispersion de nos rencontres, la multiplicité disparate des événements que nous avons vécus.

Raconter sa vie est un véritable travail: c'est accoucher de soi-même, c'est mettre de l'ordre en soi, tenter de dire qui on est en disant qui on a été et qui on voudrait être. C'est mettre en perspective des événements qui paraissent accidentels. C'est distinguer dans son passé l'essentiel de l'accessoire, les points fixes. Raconter sa vie permet de souligner des moments plus importants et aussi de minimiser d'autres événements. On peut, en effet, mettre plus ou moins de temps à raconter un événement qu'à le vivre. Pour raconter, il faut choisir ce que l'on veut mettre en évidence et ce que l'on veut mettre entre parenthèses. Le récit crée une intelligibilité, donne un sens à ce que je fais. Raconter, c'est mettre un peu d'ordre dans le désordre. Raconter sa vie, c'est un événement de la vie, c'est la vie qui se raconte elle-même pour se comprendre elle-même. La crise survient quand cette opération est devenue difficile, voire impossible. 
Mais, quand on raconte sa vie, on ne raconte pas n'importe quoi. Quand j'étais enfant, on m'a raconté ma vie à moi. Mes parents m'ont raconté ma vie en me racontant d'une certaine façon leur vie à eux. Ma vie a pris conscience d'elle-même à l'intérieur de récits qui étaient déjà enchevêtrés les uns dans les autres. Je ne raconte pas mon histoire tout seul: dans mon histoire que je vous raconte, il y a déjà l'histoire des autres.

L'histoire que nous racontons n'est évidemment pas une fiction arbitraire. Elle a un début qui est marqué par ce que les autres nous ont raconté d'eux-mêmes et de nous-mêmes. Et elle comporte aussi une "fin", une indication sur la manière de poursuivre le récit. Cette clé nous est donnée par ce que nous attendons de nousmêmes, par ce que les autres attendent de nous, par ce que les autres nous disent que nous avons à faire. Cela ne signifie évidemment pas qu'il faille toujours faire ce que l'on nous dit, mais bien que notre avenir n'est pas totalement indépendant de ce que les autres attendent de nous.

Notre récit est donc enchevêtré dans celui des autres. Et c'est ici que se noue le lien entre l'effondrement du sens dans notre société et la crise personnelle quand elle survient. Nous avons dans notre récit des évidences communes que nous partageons avec d'autres. Ainsi, dans une même famille, il y a des parties communes aux récits de ses membres. Et il en va de même dans une longue amitié. Quand on a éduqué un enfant, le récit de l'enfant et celui de son éducateur comportent une partie commune que chacun racontera à sa manière (en omettant par exemple certaines choses et en insistant sur d'autres) mais qui n'en est pas moins authentiquement partagée.

La crise est toujours une crise du sens parce qu'elle pose les questions: «À quoi suis-je fidèle? De quoi suis-je occupé à me détacher? Comment suis-je en train de me recomprendre moi-même? »Si l'on invente une histoire que l'on raconte à des enfants, on ne sait pas toujours comment on va la continuer. Cependant, on est tenu, si l'on ne veut pas mécontenter les enfants, de continuer l'histoire déjà commencée. On inventera donc une suite de jour en jour, mais en restant cohérent avec ce qu'on a dit auparavant. C'est ce que j'appelle la fidélité.

Mais la fidélité à soi-même n'est pas donnée d'emblée. Raconter l'histoire de sa vie, c'est tout autre chose que raconter une histoire à des enfants. Dans ce dernier cas, ce qui importe, c'est le suspense et les anecdotes amusantes. Dans le premier cas, ce qui est en jeu, c'est ma capacité de me faire illusion à moi-même et de raconter inconsciemment une histoire qui n'est pas vraiment la mienne. Mon histoire n'est écrite nulle part ailleurs que dans le récit que j'en fais; et cependant, si j'entends qu'elle soit l'histoire vraie de moi-même, elle doit être conforme à ma destinée secrète. Comment donc raconter en vérité l'histoire de sa vie?

C'est une question redoutable, car elle conduit droit au cœur des terreurs les plus enfouies et des espoirs les plus cachés. Il n'y a de récit vrai de ma vie que dans le récit qui se donne à interpréter à l'oreille d'un autre qui sait me désarçonner de mes propres illusions en écoutant, au travers des mots de l'histoire, la souffrance secrète qui l'habite et qui, par la vertu de son oreille, pratique en moi cette brèche par laquelle je serai délivré, petit à petit, de mes illusions les plus tenaces. Finalement, cette délivrance sera accomplie quand il s'avérera qu'elle est elle-même illusoire et que la seule chose qui compte, en définitive, c'est le mouvement du récit à la recherche de sa propre vérité.

\section{LA VÉRITÉ N’EST PAS}

UN CONTENU À DÉVERSER

DU CRUCHON

DE VOTRE SCIENCE

\section{DANS LE VERRE D'IGNORANCE}

DE VOS PATIENTS.

\section{LA VÉRITÉ, C'EST UN ÉVÉNEMENT}

QUI SURVIENT ENTRE

\section{DEUX PERSONNES}

QUI SE RISQUENT

\section{À SE PARLER AUSSI}

\section{RESPECTUEUSEMENT}

\section{QUE CLAIREMENT.}

Ce sont d'ailleurs les impasses du récit, ses blocages, ses redites qui constitueront pour l'oreille attentive les repères les moins fragiles de l'itinéraire qui tente de venir au jour par le truchement de ces mots qui en vérité disent à la fois plus et moins que ce qu'ils disent en apparence.

Mais, dans le récit d'une vie, il y a des choses fixes, importantes, et d'autres qui sont mobiles. Il faut donc distinguer deux niveaux de crise. Il y a des crises qui sont superficielles parce qu'elles sont à la surface: il s'agit d'une simple difficulté de continuer à raconter, mais non d'une impossibilité de raconter. Des crises plus profondes surgissent quand on ne peut vraiment plus continuer à raconter l'histoire, à moins de renverser la situation. Une redistribution des rôles peut alors s'imposer et un nouvel acteur peut surgir. Cette redistribution ne change pas les chapitres précédents de l'histoire (parce qu'on ne vit qu'une fois et que l'on n'a qu'une vie à raconter), mais elle change le sens des chapitres précédents. Il faut parfois réinventer un sens à son passé pour pouvoir affronter créativement l'avenir. Ce sont les crises les plus profondes. Je pense que les psychologues et les psychiatres ne me contrediront pas. Une psychothérapie consiste d'ailleurs à raconter et à inventer (créer et trouver) une harmonie nouvelle qui remette en place une série de fragments qui sont défaits et déjetés çà et là.

Pour surmonter une crise, il faut pouvoir transgresser la logique des chapitres précédents de l'histoire, être capable d'un renversement de situation. Ainsi, par exemple, si l'on cherche la logique de l'histoire au fur et à mesure qu'on lit un roman policier (sans jamais sauter à la dernière page pour savoir la fin de l'histoire avant de l'avoir parcourue entièrement), on fait des hypothèses sur le coupable. Mais subitement, de temps à autre, surviennent des éléments qui bouleversent complètement toutes les hypothèses en cours. Que faire sinon le deuil des anciennes hypothèses? Il n'y a pas d'autre issue que de reconnaître qu'on s'est fourvoyé et de chercher un nouveau schéma d'interprétation des données connues. Et, si le livre est bien fait, ce type de réajustement sera nécessaire jusqu'à la dernière page. Se défaire de l'ancienne compréhension est pénible, car c'est se séparer de quelque chose qu'on a soi-même construit. C'est cependant la seule issue créative possible.

On peut être dérouté dans sa propre vie, mais aussi dérouter ceux à qui on la raconte. C'est ce qui se passe pour quelqu'un dont on dit parfois qu'il «refait sa vie». La crise est donc une situation où mon identité personnelle profonde est en jeu: je me demande qui je suis au juste, je ne le sais plus très bien et j'ai à me (ré)inventer en tâchant d'éviter les ornières du récit dont j'ai à me départir parce qu'il m'enferme dans une fallacieuse intelligibilité de la réalité. Ce qui est habileté de l'écrivain pour le plaisir du lecteur dans le cas du roman policier est enjeu vital pour celui qui tente d'être fidèle à ce qu'il devient. Et l'enjeu vital, c'est toujours la mort, car la mort est liée inexorablement au changement. C'est d'ailleurs pourquoi la crise est une occasion de changement.

Tout change. Déjà les plus vieux des philosophes grecs disaient que tout passe, tout coule, tout change. Le temps passe, nous allons tous vers la mort tandis que d'autres vies surgissent. Certes, un 
changement perpétuel s'opère naturellement dans le cours des choses, les saisons se succèdent, la vie intérieure évolue, les relations avec autrui se modifient. Mais le changement le plus radical survient dans la parole échangée avec autrui qui, seul, peut m'empêcher de pratiquer à l'égard du changement la politique de l'autruche qui me maintient dans l'illusion de la stabilité. Tout change et je résiste au changement parce que secrètement il implique la mort. Le temps qui passe m'entraîne à la mort et, dans ces conditions, je n'ai pas envie de voir où je vais.

Le changement suscite de multiples résistances. Il y a des résistances à l'intérieur de nous, car nous avons peur de savoir qui nous sommes en vérité. Mais il y a aussi des résistances très fortes autour de nous. Tout se passe comme s'il fallait copier des modèles, c'est-à-dire, précisément, ne pas changer mais reproduire des stéréotypes, répéter des situations bloquées et bloquantes. Mais les stratégies de résistance sont multiples: ce peut être également d'imputer la crise à la situation sociale ou à d'autres, se déchargeant ainsi sur des boucs émissaires de la véritable question qu'on est pour soi-même. Ce sont les crises mortnées, celles qui avortent parce que tout est disposé autour de ceux qui les vivent pour qu'elles soient déniées et ne viennent pas au langage. Réprimer les crises successives que comporte inévitablement une existence humaine, c'est la précipiter vers une mort plus terrible que la mort, c'est la faire mourir avant même que de la laisser naître.

Il faut bien reconnaître d'ailleurs que la médecine comme phénomène social de normalisation des déviances sociales peut apparaître dans nombre de cas et de situations comme une instance destinée à refouler l'interrogation fondamentale de l'être humain affronté à la question de la vérité de son existence. Vouloir à tout prix mettre au jour la cause organique d'un malaise «métaphysique» est une attitude qui, pour n'être sans doute ni délibérée ni même consciente, n'en est pas moins meurtrière. On touche ici à une nouvelle figure de la contradiction médicale: la médecine refoule en nous la question qu'elle-même nous pose avec le plus d'acuité en nous donnant le pouvoir de nous transformer nous-mêmes. Il y a donc des stratégies à inventer pour déjouer les résistances soulevées par la perspective du changement et faire d'une crise un événement vivifiant plutôt qu'un processus mortifère.

Quelles conditions devraient être remplies pour qu'une crise soit une occasion de changement? En son principe, la réponse est simple: il faut que la crise vienne au langage. Il y a des crises stériles et des crises fécondes. Il y a des crises subies et des crises assumées. Il y a des crises réprimées, passées sous silence et des crises qui viennent au langage et qui, de ce fait, sont parfois surmontées.

Pour qu'une crise puisse ouvrir vers un changement, pour qu'une maladie puisse devenir une expérience positive, il faut que j'arrive, avec l'aide du médecin ou d'autres personnes, à trouver en moi-même la force de la vaincre. La première condition est donc d'avoir encore un mécanisme de défense pour pouvoir exercer une créativité fidèle. La fidélité, c'est continuer l'histoire; la créativité, c'est inventer la suite. Pour surmonter sa crise, il faut être capable de penser à soi-même comme à quelqu'un qui est capable d'inventer une suite à sa propre histoire.

Mais on ne raconte pas une histoire à des murs, on la raconte à quelqu'un. Et la condition la plus essentielle pour qu'une crise soit une occasion de changement, c'est précisément que quelqu'un soit là pour écouter l'autre qui est en crise et qui se raconte, écouter sans banaliser, écouter sans juger, attentif à saisir avec lui le sens de sa propre histoire.

La troisième condition pour qu'une crise soit occasion de changement est que l'on puisse comprendre son passé (toutes les conditions se tiennent) de façon à le réouvrir à un avenir. Et cela implique bien entendu que l'on reconnaisse la crise pour ce qu'elle est et que l'on ne se laisse pas entraîner à suivre la politique de l'autruche. Il s'agit de reconnaître que quelque chose a été perdu irrémédiablement et que cela ne peut plus être désormais qu'un souvenir. Il s'agit, en quelque sorte, de faire son deuil d'une flatteuse mais fallacieuse image de soi-même. Cela ne veut pas dire qu'il faut être brutal avec soi-même mais lucide, car si l'on n'est pas clairement conscient qu'on est en crise, comment pourrait-on en sortir?

La crise est un moment dramatique: un moment de jugement, de réévaluation des enjeux de la vie, de restructuration de la hiérarchie des valeurs. C'est un moment «critique» au sens étymologique du mot, c'est-à-dire un moment de discernement. Et celui, médecin, psychologue ou simple «semblable», qui est témoin attentif de la crise peut dédramatiser sans banaliser: il ne s'agit pas d'expliquer en quel point exactement l'être-en-crise se trouve sur telle ou telle courbe de Gauss, mais bien plutôt de lui proposer l'hypothèse et la conviction qu'il y a assez de points d'appui à l'intérieur de lui-même et de ressources inexplorées dans les chapitres précédents de son histoire pour qu'il soit raisonnable d'imaginer une suite positive.

Personne n'a jamais fini de changer et personne n'est jamais à l'abri d'une crise. La dernière crise, c'est la mort. Et ce sont les réactions aux crises qui font les grandes étapes de la vie, qui préparent à être solide ou fragile, à être disponible ou réfractaire au jour de la mort.

La crise ne sera occasion de changement que si l'on est déjà de plain-pied dans le changement et la créativité. Écouter l'autre en crise, c'est peut-être accepter d'entrer sur la pointe des pieds dans la conversation intérieure qu'il nous confie pour un moment; et, après avoir glissé quelques mots dans cette conversation pour lui redonner sa vitalité, c'est sans doute aussi se retirer de cette conversation comme on y est entré: sur la pointe des pieds.

C'est à ce prix et à ce prix seulement que la médecine évitera de devenir chaque jour davantage cette machine de normalisation sociale que souligne l'analyse sociologique qui sera évoquée plus loin. C'est au prix de cette écoute qui n'a rien d'organique, bien qu'elle n'exclut pas a priori l'existence d'une altération organique, que le médecin peut remplir sa véritable fonction sociale qui est d'abord de ne pas nuire, c'est-à-dire de ne pas enrôler dans la maladie ceux qui n'ont pas à l'être, ensuite de diagnostiquer la maladie de ceux qui souffrent d'altérations organiques ou fonctionnelles et, enfin, de les aider à recouvrer la santé.

C'est à ce prix et à ce prix seulement que la médecine entendra la souffrance des hommes au lieu de la faire taire.

\section{Bibliographie}

«Ethica clinica» (Revue) (1999). Numéro sur les soins palliatifs intitulé L’impossible décision, Namur.

ARISTOTE (éditions diverses). Éthique à Nicomaque, Paris, Librairie philosophique J. Vrin.

MALHERBE, J.-F. (1987). Pour une éthique de la médecine, Paris, Larousse; (1997), 3 édition augmentée, collection "Catalyses", Montréal, Éditions Fides (diffusion en France par les Éditions du Cerf à Paris).

MALHERBE, J.-F. (1996). Homicide et compassion. L'euthanasie en éthique clinique, Montréal, Éditions Médiaspaul (diffusion en France par les Éditions St-Paul à Paris).

MALHERBE, J.-F. (2003a). Les Ruses de la violence dans les arts du soin, Montréal, Éditions Liber.

MALHERBE, J.-F. (2003b). Le Cri de Dolorès. Penser la violence dans les relations d'aide, Sherbrooke, Éditions GGC.

\section{Note}

1. Ce texte est une version abrégée et remaniée de la transcription d'une conférence donnée à Poitiers le 20 mai 2000 et publiée sous le titre "Afin que nul ne meure avant que d'être né: Esquisse d'une philosophie des soins palliatifs », dans René ROBERT (2000). Éthique en réanimation, Société de réanimation de langue française, Actes du Colloque de Poitiers, 19-20 mai 2000, Paris, Elsevier, p. 39-48. 